4. Katzenstein H. Hepatocellular Carcinoma in Children and Adolescents: Results From the Pediatric Oncology Group and the Children's Cancer Group Intergroup Study. J Clin Oncol. 2002;20(12):2789-2797.

5. McKiernan P. Nitisinone in the Treatment of Hereditary Tyrosinaemia Type 1. Drugs. 2006;66(6):743-750.

\section{Successful treatment of refractory external pancreatic fistula by transpapillary stent insertion into the fistula tract (endoscopic fistuloduodenostomy)}

Management of external pancreatic fistulae (EPF) resulting from iatrogenic percutaneous drainage of acute pancreatic collections is difficult. ${ }^{1}$ The outcome is good when transpapillary insertion of endoprosthesis is able to completely bridge the disruption of the pancreatic duct. ${ }^{2}$ However, when bridging of disruption is not achieved the EPFs are difficult to treat. While percutaneous modalities have been used previously to place stents into the fistula tract, ${ }^{3}$ we describe a case of 55 year old lady who was treated with endoscopic transpapillary placement of stent into the fistula tract thereby creating an endoscopic fistuloduodenostomy.

\section{Case Report}

A 55 year old female underwent percutaneous drainage (PCD) for infected acute necrotic collection. She was a known case of chronic kidney disease and had gall stone related acute necrotising pancreatitis three weeks ago. Following PCD her symptoms improved and purulent discharge was gradually replaced by $300-500 \mathrm{ml}$ of clear pancreatic juice. As the discharge from the fistula continued unabated for 4 weeks, patient was taken up for endoscopic retrograde pancreatography (ERP). On ERP, partial duct disruption was seen at neck of pancreas (Figure 1) with fistula tract being also delineated (Figure 1 arrow). On repeated attempts guide wire could not be negotiated upstream to the disruption and therefore 5Fr stent was placed in the disruption. As there was no decrease in output patient was taken up for repeat ERP. In spite of repeated attempts the duct disruption could not be bridged and therefore guide wire was taken into the fistula tract. As the PCD catheter was blocking the guide wire, it was withdrawn and fistula tract clearly delineated (Figure 2). Thereafter, deep cannulation of the fistula tract was achieved and 5Fr transpapillary stent placed into the fistula tract (Figure 3). Following this the PCD catheter was removed and the external pancreatic fistula (EPF) healed. Follow up, computed tomography (CT) of the abdomen did not reveal any residual collection and the stent was seen in situ (Figure 4). The patient was referred to surgical services for cholecystectomy.

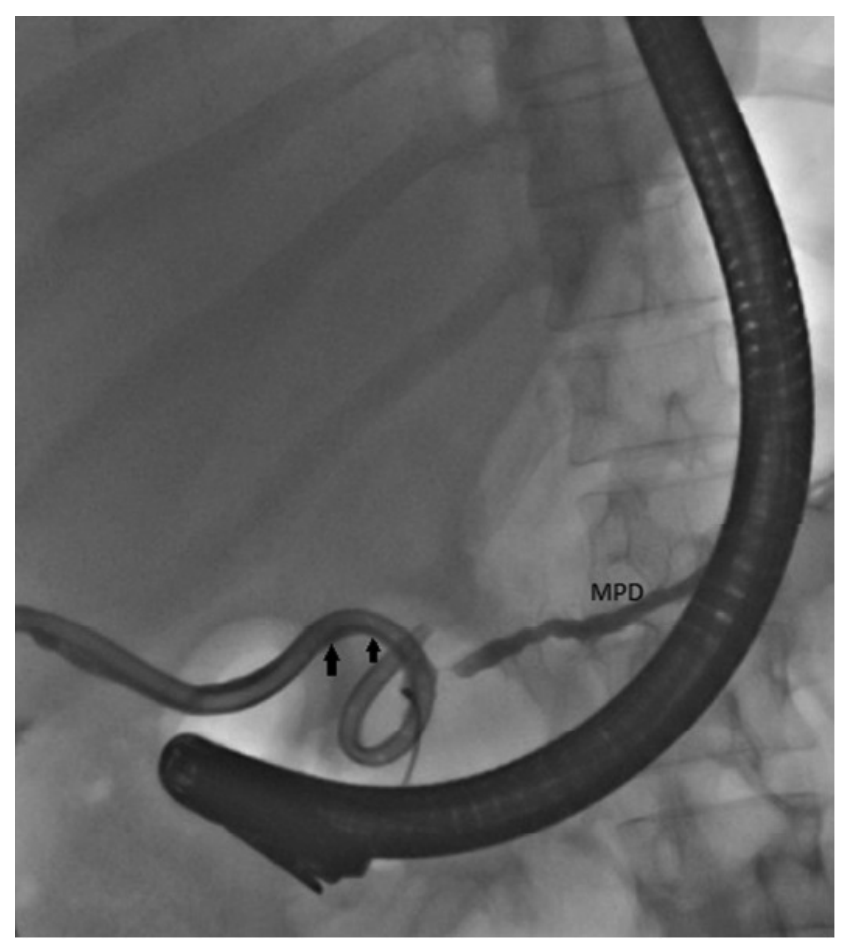

Figure 1: ERP: Partial duct disruption with upstream pancreatic duct (MPD) and fistula tract with catheter (arrow). 


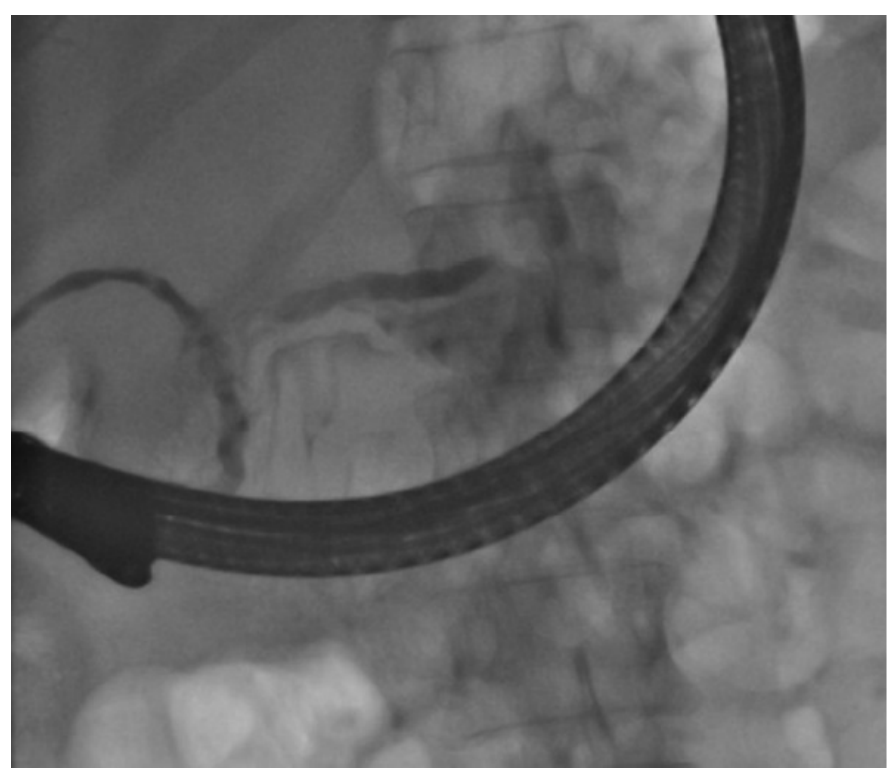

Figure 2: ERP: PCD withdrawn and fistula tract clearly delineated.

\section{Discussion}

Pancreatic duct disruption can result from acute or chronic pancreatitis or pancreatic trauma. The leakage of pancreatic juice from the disruption can lead to local complications like acute pancreatic fluid collection, walled off necrosis or pancreatic pseudocysts, pancreatic pleural effusion or ascites. ${ }^{1}$ Use of percutaneous drainage catheters to drain infected or symptomatic fluid collections may convert an internal pancreatic fistula into an external pancreatic fisula. ${ }^{2}$ Pancreatic fistulae from large disruptions are unlikely to heal spontaneously and require treatment. On ERP, pancreatic duct disruption is usually identified by leakage of contrast out of the ductal system. The disruption is called a complete one if the upstream pancreatic duct (PD) is not opacified and is termed partial if the upstream PD is opacified by the contrast. ${ }^{1}$ While transmural drainage of walled off collections is possible by direct endoscopic or transmural drainage, in situations where an iatrogenic EPF has been created the transmural drainage is not feasible. ${ }^{2}$ In these situation a transpapillary drainage with the aim of bridging the pancreatic duct disruption with an endo-prosthesis is the preferred therapeutic approach. The best clinical outcomes are obtained when the duct disruption is partial or when the stent can bridge the disruption. ${ }^{1,2}$ When the disruption

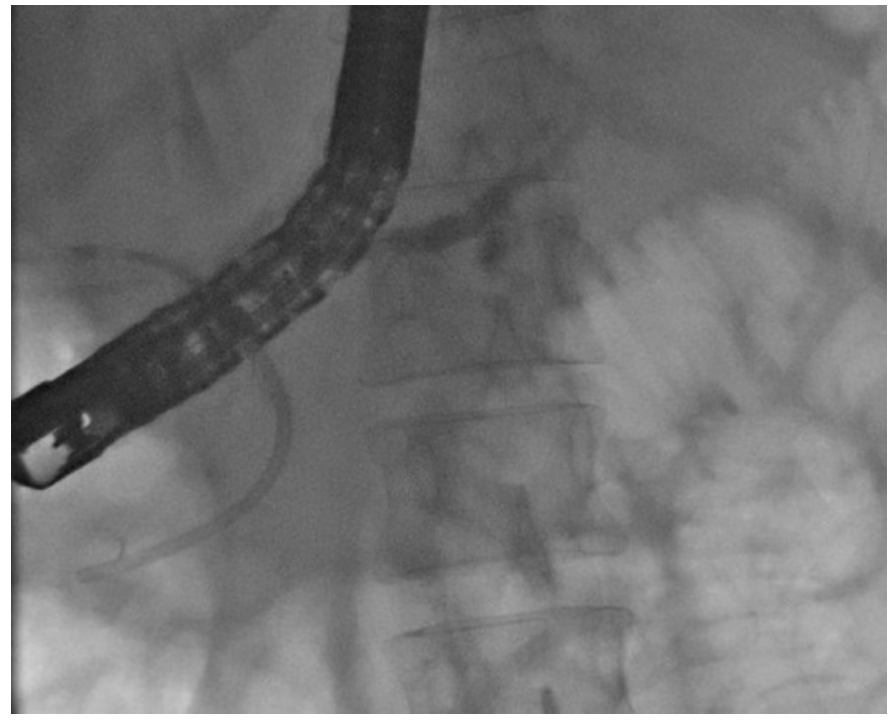

Figure 3: Transpapillary stent placed in fistula.

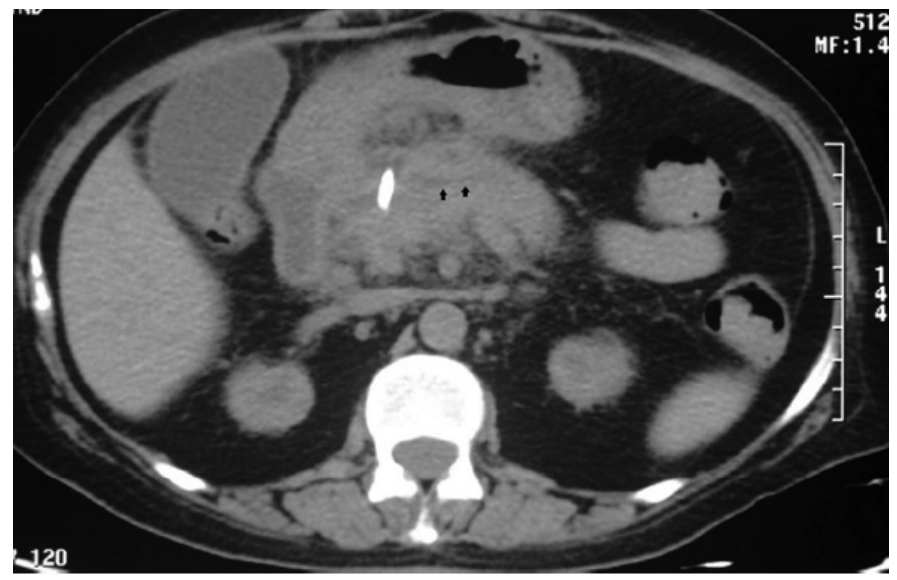

Figure 4: CT abdomen: Stent tip seen at mouth of fistula. Upstream duct can also be seen (arrows).

cannot be bridged, however, it is usual to place the stent into the disruption in hope of achieving an adequate internal drainage. Clinical outcomes are not good if the disruption cannot be bridged as in complete disruption. In such situation, also termed as disconnected pancreatic duct syndrome, transmural drainage is required which is not feasible with use of EUS or ERCP due to creation of external pancreatic fistula. Previously, some workers have described a rendezvous approach in such difficult cases wherein the percutaneous drainage site was punctured into the gastrointestinal lumen (stomach/duodenum) and the guidewires were captured endoscopically to create a 
transenetric fistula and stents were placed into the fistula tract close to the pancreatic duct disruption. ${ }^{3}$ Occasionally the percutaneous route of drainage may be through a gastrointestinal organs (eg stomach) and this tract can also be used to create an internal drainage to treat EPF. ${ }^{4}$ In the present case, we have accomplished the drainage of the fistula tract through a transpapillary stent instead of a percutaneous approach. Thereby, we were able to drain the pancreatic juice from the disruption intro the duodenum through a transpapillary stent. This, to the best of our knowledge, is the first report of an endoscopic fistuloduodenostomy to manage an external pancreatic fistula.

\section{SURINDER SINGH RANA VISHAL SHARMA \\ RAVI SHARMA LOVNEET DHALARIA PUNEET CHHABRA RAJESH GUPTA DEEPAK K BHASIN}

Departments of Gastroenterology \& Surgery, Post Graduate Institute of Medical Education and Research (PGIMER), Sector 12, Chandigarh-160012, India.

Correspondence: Surinder Singh Rana Email:drsurinderrana@yahoo.co.in

\section{References}

1. Varadarajulu S, Rana SS, Bhasin DK. Endoscopic therapy for pancreatic duct leaks and disruptions. Gastrointest Endosc Clin N Am. 2013;23(4):863-92.

2. Rana SS, Bhasin DK, Nanda $M$, et al. Endoscopic transpapillary drainage for external fistulas developing after surgical or radiological pancreatic interventions. J Gastroenterol Hepatol. 2010;25:1087-92.

3. Irani S, Gluck M, Ross A, et al. Resolving external pancreatic fistulas in patients with disconnected pancreatic duct syndrome: using rendezvous techniques to avoid surgery (with video). Gastrointest Endosc. 2012;76:58693.

4. Rana SS, Sharma V, Bhasin DK. External pancreatic fistula with disconnected pancreatic duct treated by transmural stent insertion through the transgastric percutaneous catheter site. Gastrointest Endosc. 2015;82:1130-2. 